\title{
A Kinetic Model for Tribological Surface Transformations Occuring on the Railroads: Irreversible Near-Surface Metallurgical Transformations
}

\author{
G. Antoni \\ LMA UPR 7051, CNRS, Aix-Marseille Université, 13331 Marseille Cedex 03, France
}

Correspondence should be addressed to G. Antoni, antoni@lma.cnrs-mrs.fr

Received 19 July 2012; Accepted 9 September 2012

Academic Editors: A. Chrysanthou, C. Panagopoulos, and L. Takacs

Copyright (c) 2012 G. Antoni. This is an open access article distributed under the Creative Commons Attribution License, which permits unrestricted use, distribution, and reproduction in any medium, provided the original work is properly cited.

\begin{abstract}
During the manufacturing processes or the operational phases, some materials are liable to undergo metallurgical phenomena known as irreversible solid-solid phase transformations or Tribological Surface Transformations (TSTs). The treads of several rails in the French railroad network have been affected by TSTs. The kinetic model presented in this paper describes the initiation of TSTs and their development near the rail surface.
\end{abstract}

\section{Introduction}

For the last twenty years, the weight and velocity of freight trains have been increasing steadily, resulting in the development of "Tribological Surface Transformations" (TSTs) or "White Etching Layers" (WELs) [1-4] (see Figure 1(a)). TSTs are the irreversible solid-solid phase transformations which occur both at the surface and in depth, in the immediately vicinity of the wheels of passing trains. These processses, which mainly occur in straight parts of the rails, are induced by heavy corrugation [5-7] (see Figure 2), which generates an alternating pattern of troughs, consisting of the initial material (ferrito-pearlitic steel) and crests, forming the damaged part, where the material is transformed into "quasi-martensitic" steel (see Figure 1(b)) at depths ranging from several nanometers to more than $100 \mu \mathrm{m}$ [8]. The values obtained in microhardness tests on TSTs in steel rails generally range from 700 to $1000 \mathrm{H}_{v}$ (corresponding to a martensitic phase), as compared with the initial hardness of ferrito-pearlitic rails, which is about $300 \mathrm{H}_{v}$ [9]. Rails with TSTs may, in the worst cases, develop cracks due to strong strain incompatibilities between the hard "white" phase (martensite) and the soft "untransformed" phase (ferritepearlite), see Figure 3 [10]. Although the origin of TSTs has not yet been clearly established, it seems to involve contact fatigue processes $[11,12]$. The repeated mechanical loads applied (normal and shear stresses) combined with the highly localized thermal effects occurring near the surface at the wheel/rail contact points as the result of the friction may be largely responsible for these transformations [13].

Based on the above assumption, the kinetic model for TSTs [14] presented in Section 2 was developed. This model takes the thermo-mechanical state in the close vicinity of the wheel/rail contact area into account, that is, the fact that the high mechanical stress levels and the local temperature increase occurring near the surface may generate irreversible "quasi-surface" solid-solid phase transformations.

\section{Metallurgical Behavior Model for the Irreversible Solid-Solid Phase Transformations and TSTs}

In line with the principles of causality, whereby the future cannot influence the current state and those of localization, whereby the state of a material point depends only on its past history, and not on that of the neighboring material points, the metallurgical state of the material point $X$ at a given time $t$ is a function of both the temperature and the strain history, that is,

$$
z(X, t)=\underset{\tau \leq t}{\mathcal{F}}(T(X, \tau), \boldsymbol{\epsilon}(X, \tau)),
$$




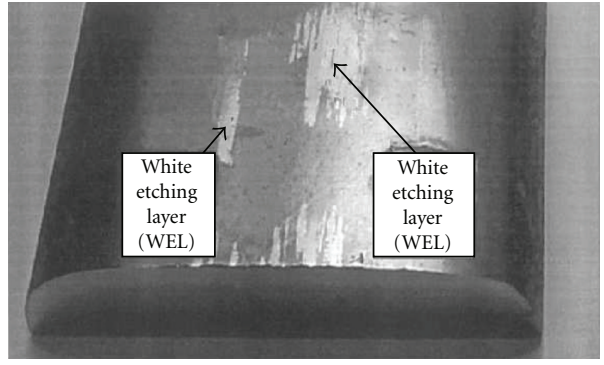

(a)

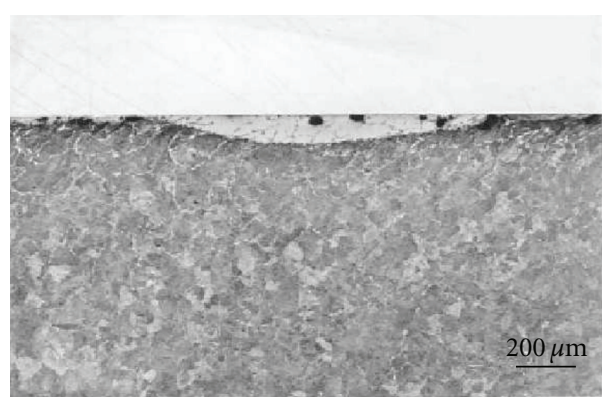

(b)

FIGURE 1: (a) Macroscopic view on the tread of a rail (S54/900A steel) showing TSTs or "White Etching Layer". (b) Transverse cross-section of the upper part of a rail (a). The parent phase (grey part) is ferrite pearlite, which still remains in the rail when TSTs have developped and the daughter phase (white part), occurring at the surface, is "quasi-martensite" (extract from [8]).

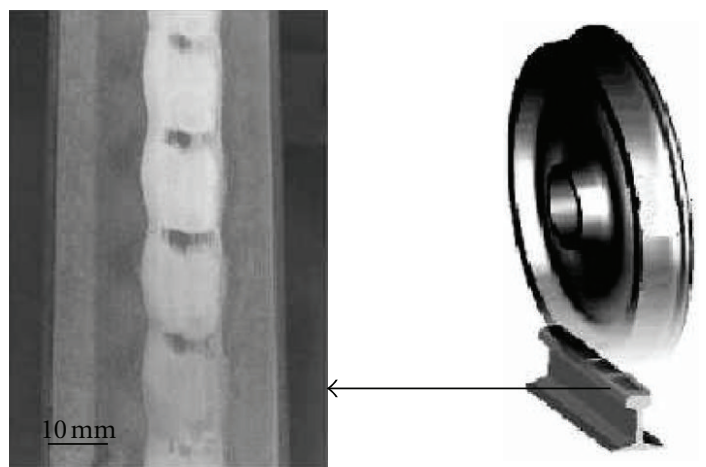

FIGURE 2: Rail corrugation: alternating of the troughs (grey part: ferrito-pearlitic phase) and crests (white part: "quasi-martensitic" phase) (extract from [7]).

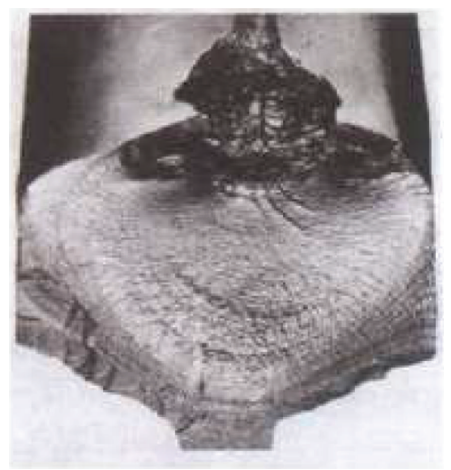

Figure 3: Fatigue defect due to wheel/rail contact: the "Squat" which corresponds to the local cracking and sagging of the rail tread. This defect occurs mainly in straight parts of railway tracks (extract from [10]).

where $z \in[0,1]$ is the metallurgical variable, $\mathcal{F}$ denotes the metallurgical functional, $T$ and $\boldsymbol{\epsilon}$ are the temperature and strain tensor, respectively. Note that when $z=0$ (resp., $z=1$ ), the material is composed of a single phase, that is, a ferrite-pearlite phase (resp., a martensite phase), and when $z \in] 0,1[$, it consists of two phases.
In the framework of Continuum Thermodynamics with internal variables [15], this functional dependence can be overcome by replacing the memory effects by additional internal variables. We assume that the equation of evolution of the metallurgical variable $z$ can be written as

$$
\dot{z}=f(\&, \dot{T}, \dot{\boldsymbol{\epsilon}}, \nabla T),
$$

where $\&=\left(T, \boldsymbol{\epsilon}, \boldsymbol{\sigma}, z, \vartheta^{i}\right)$ denotes the thermomechanical state of the material point $X$ at a given time $t, \sigma$ is the stress tensor and $\vartheta^{i}$ are the additional internal variables (for example, the martensitic grain size); in a first approximation, $\vartheta^{i}$ are not taken account that is, $\vartheta^{i}=0$.

The following kinetic model is adopted in order to describe the irreversible solid-solid phase transformations involved in TSTs:

$$
\dot{z}=\kappa \dot{p}
$$

with $\dot{p}=\frac{\left\langle\sigma^{\mathrm{eq}}+\delta P-\left(\sigma_{y}(T, \mathbf{q})+\beta(z / \kappa)\right)\right\rangle}{\bar{\sigma}_{y} \eta} H(1-z) \geq 0$,

$$
\sigma_{y}(T, \mathbf{q})=\left\{\begin{array}{l}
\bar{\sigma}_{y} \exp \left(\frac{T_{i}-T}{T_{i}}\right) \\
\text { or } \\
\bar{\sigma}_{y} \exp \left(-\frac{\|\mathbf{q}\|}{\left\|\mathbf{q}_{c}\right\|}\right)
\end{array}\right.
$$

where $\bar{\sigma}_{y}$ denotes the TST's yield strength when $T=T_{i}$ (or $\mathbf{q}=0), T_{i}$ is the initial temperature, $\sigma^{\mathrm{eq}}=((3 / 2) \mathbf{s}: \mathbf{s})^{1 / 2}$ is the Von Mises equivalent stress, $\mathbf{s}$ is the deviatoric stress tensor, $P=-(1 / 3) \operatorname{tr}(\boldsymbol{\sigma})$ is the pressure, $\sigma$ is the Cauchy stress tensor, $\delta$ is a material parameter associated with pressure sensitivity, $\mathbf{q}=-k \nabla T$ is the heat flux (Fourier's law), $k$ is the thermal conductivity, $\mathbf{q}_{c}$ is the critical heat flux, $\kappa$ and $\beta$ are material parameters associated with the irreversible solid-solid phase transformation, $\langle\cdot\rangle$ denotes the Macaulay brackets $(\langle x\rangle=x$ when $x \geq 0$ and $\langle x\rangle=0$ when $x<0), H(\cdot)$ denotes the Heaviside step function $(H(x)=1$ when $x \geq 0$ and $H(x)=0$ when $x<0), \eta$ is the characteristic 
time of the viscous effets associated with the TST process, and $\|\cdot\|$ is the Euclidean norm.

Note that: (i) Equation (3b) shows that the irreversible solid-solid phase transformation (or TST) is activated by the normal and shear stresses and accelerated with the temperature. The transformation occurs at stress levels which trigger dislocation motions due to the strain incompatibilities between the ferrite-pearlite and martensite phases. The triggering of these dislocation motions is facilitated by the increase in temperature at a given stress state. (ii) The irreversibility of the solid-solid phase transformation is expressed by $\dot{z}$, which cannot be negative. In addition, the eligibility field of the metallurgical variable $z \in[0,1]$ is also confirmed. (iii) Equation (3c) means that the TST's yield strength depends on either the temperature $(T)$ or its gradiant $(\nabla T)$. When $T \geq T_{i}$ (or $\left.\mathbf{q} \geq \mathbf{q}_{c}\right)$ in the immediate vicinity where the thermomechanical load is applied, the area is "thermally activated" and the original, nontransformed material $\left(z_{0}=0\right)$ undergoes an irreversible solid-solid phase transformation at stress levels which are lower than the TST's yield strength $\bar{\sigma}_{y}$.

\section{An Illustrative Example}

The initiation and development of TSTs occur during the complex processes at work in a rail when a train is crossing over it: this situation is also known as the wheel/rail problem (see Figure 4). The repeated mechanical loading with friction applied to the rail due to the passing of trains creates a TST thickness $\left(h_{\mathrm{TST}}\right)$ in the immediate vicinity of the wheel/rail contact area, whereas the basic material constituting the rail remains unchanged beyond this area. In this section, the case of the structure studied does not resemble the wheel/rail problem, it follows therefore that the nonlinear contact and friction between wheel and rail are not taken into account. It is proposed here to solve a $1 \mathrm{D}$ problem by examining the rail in depth in order to confirm that the kinetic model presented in Section 2 can generate irreversible solid-solid transformations near the surface at which the thermomechanical loads are applied.

Taking the case of small perturbations, a thermoelastic bar (see Figure 5(a)) liable to undergo irreversible solid-solid transformations is subjected to quasi-static thermomechanical loading conditions, where the lateral surfaces of the bar are taken to be adiabatic in a first approximation.

The following constitutive equations are used for this purpose:

$$
\begin{gathered}
\sigma=E\left(\epsilon-\alpha\left(T-T_{i}\right)-\frac{z}{\kappa} \operatorname{sgn}(\sigma)\right), \\
\dot{z}=\kappa \frac{\left\langle|\sigma|-\left(\sigma_{y}(T, \mathbf{q})+\beta(z / \kappa)\right)\right\rangle}{\bar{\sigma}_{y} \eta} H(1-z), \\
\sigma_{y}(T, \mathbf{q})=\left\{\begin{array}{l}
\sigma_{y}^{1}=\bar{\sigma}_{y} \exp \left(\frac{T_{i}-T}{T_{i}}\right) \\
\text { or } \\
\sigma_{y}^{2}=\bar{\sigma}_{y} \exp \left(-\frac{\|\mathbf{q}\|}{\left\|\mathbf{q}_{c}\right\|}\right),
\end{array}\right.
\end{gathered}
$$

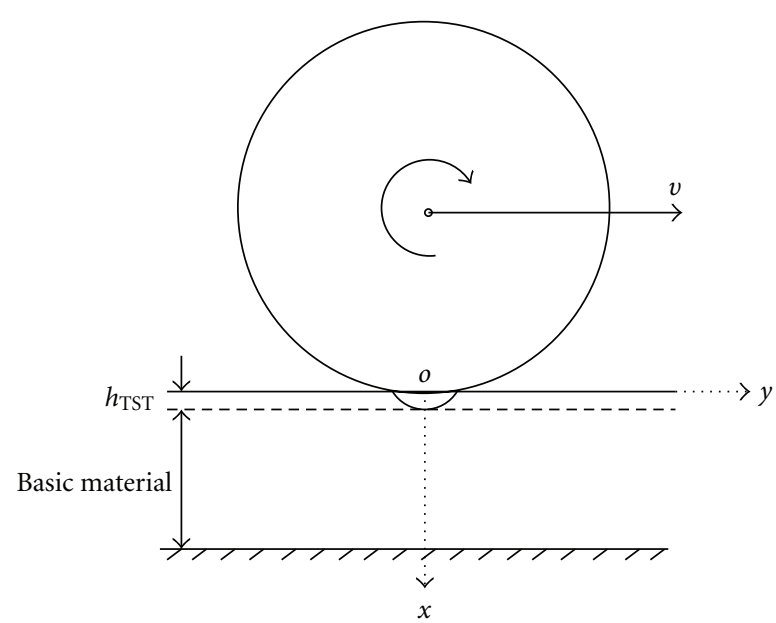

FIgURE 4: Wheel/rail problem with TSTs: presence of a TST thickness, $h_{\mathrm{TST}}$ (martensite), in the close vicinity of the wheel/rail contact area, below the basic material (ferrite pearlite) of the rail.

where $E$ is the Young's modulus, $\alpha$ is the thermal expansion coefficient, $\operatorname{sgn}(\cdot)$ denotes the sign function $(\operatorname{sgn}(x)=-1$ when $x<0, \operatorname{sgn}(x)=0$ when $x=0$ and $\operatorname{sgn}(x)=1$ when $x>0$ ). Note that (4) represents the thermoelastic mechanical behavior of the bar under the assumptions mentioned above.

Assuming no inertia and zero gravity, the quasi-static equilibrium can be written:

$$
\frac{\partial \sigma}{\partial x}=0,
$$

where $\partial(\cdot) / \partial x$ denotes the first derivative with respect to $x$, $|\cdot|$ is the absolute value function $(|x|=x$ when $x \geq 0$ and $|x|=-x$ when $x<0$ ).

Therefore (7) means that the stress field $\sigma(x, t)$ is uniform and equal to the mechanical loading applied $\sigma_{0}(t)$ throughout the bar for all $x \in[0, L]$.

Neglecting the source terms in the heat equation corresponding to the thermoelasticity and solid-solid phase transformations, this reduces to

$$
\rho C_{\epsilon} \frac{T}{T_{i}} \frac{\partial T}{\partial t}-k \frac{\partial^{2} T}{\partial x^{2}}=0,
$$

where $\rho$ is the mass density, $C_{\epsilon}$ is the specific heat capacity, $\partial(\cdot) / \partial t$ and $\partial^{2}(\cdot) / \partial x^{2}$ denote the first and second partial derivatives with respect to $t$ and $x$ (temporal and spatial quantities).

The boundary conditions are summarized in Figure 5(b) and the constitutive thermomechanical parameters used are $C_{\epsilon}=450 \mathrm{~J} \cdot \mathrm{kg}^{-1} \cdot \mathrm{K}^{-1} ; E=2 \times 10^{5} \mathrm{MPa} ; \rho=78 \times 10^{-7} \mathrm{~kg}$. $\mathrm{mm}^{-3} ; \alpha=12 \times 10^{-6} \mathrm{~K}^{-1} ; \kappa=10^{-2} ; \bar{\sigma}_{y}=500 \mathrm{MPa} ; k=$ $5 \times 10^{-2} \mathrm{~W} \cdot \mathrm{mm}^{-1} \cdot \mathrm{K}^{-1} ; \eta=10^{3} \mathrm{~s} ; \beta=5 \times 10^{3} \mathrm{MPa} ; q_{c}=$ $19 \mathrm{~W} \cdot \mathrm{mm}^{-2}$.

The distribution (resp., temporal evolution) of the metallurgical variable $z$ is plotted in Figure 6(a) (resp., Figure 6(b)). We can see from $z$-distribution obtained with both values of $\sigma_{y}(T, \mathbf{q})\left(\sigma_{y}^{1}\right.$ and $\left.\sigma_{y}^{2}\right)$ that the maximum phase transformation occurs near the surface and in its immediate 


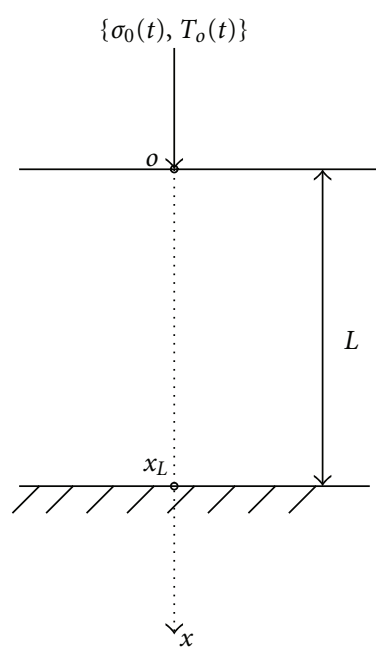

(a)

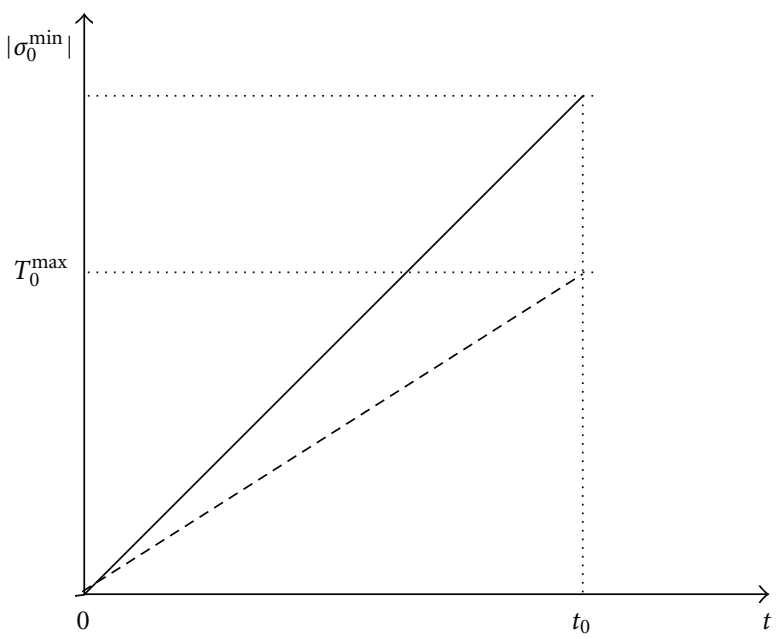

(b)

FIGURE 5: (a) 1D problem of TSTs: a bar subjected to a thermomechanical loading $\left\{\sigma_{0}(t), T_{0}(t)\right\}$ (where $L=10 \mathrm{~mm}$ ). (b) Boundary conditions: the loading function $\sigma_{0}(t)$ (resp., $T_{0}(t)$ ) is represented in solid lines with $\left|\sigma_{0}^{\min }\right|=400 \mathrm{MPa}$ at time $t_{0}=10^{-1} \mathrm{~s}$ (resp., dashed lines, with $T_{0}^{\max }=450 \mathrm{~K}$ ); At the point $x=x_{L}=L$, the temperature and the displacement are $T_{L}=T_{i}=300 \mathrm{~K}$ and $u_{L}=0$, respectively.

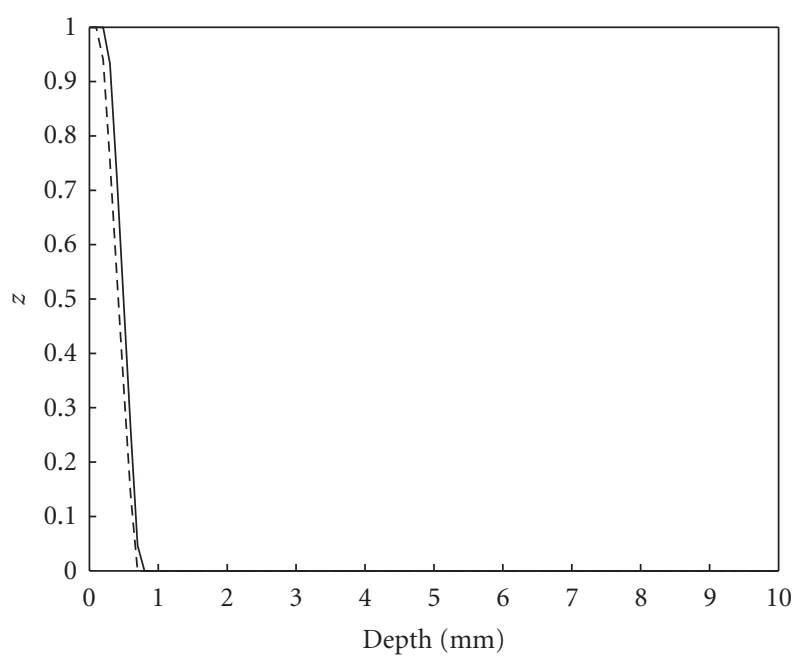

(a)

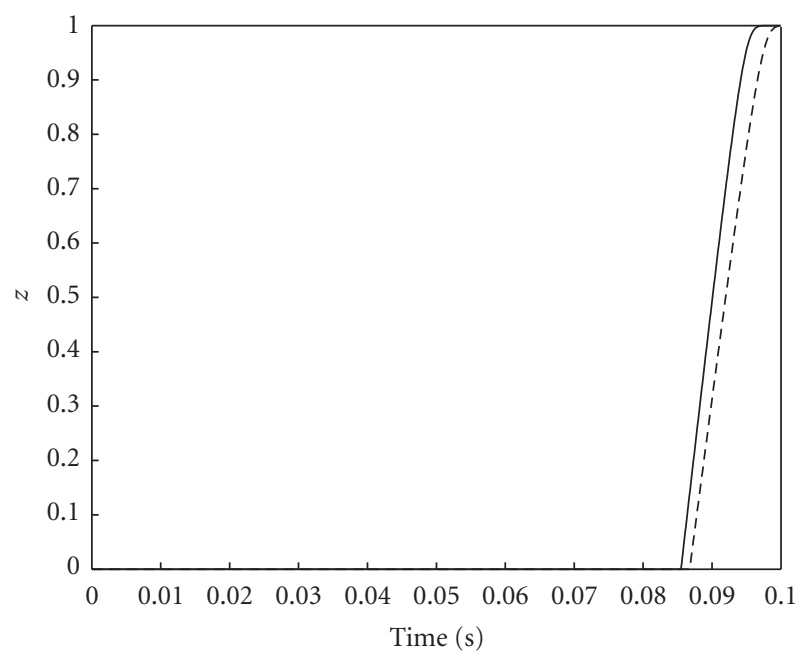

(b)

Figure 6: (a) Distribution of the metallurgical variable $z$ in the bar at time $t=t_{0}=10^{-1}$ s where $\sigma_{y}(T, \mathbf{q})=\sigma_{y}^{1}($ solid line $)$ and $\sigma_{y}(T, \mathbf{q})=\sigma_{y}^{2}$ (dashed line). (b) Temporal evolution of the metallurgical variable $z$ in the time $t \in\left[0, t_{0}\right]$ where $\sigma_{y}(T, \mathbf{q})=\sigma_{y}^{1}($ solid line $)$ and $\sigma_{y}(T, \mathbf{q})=\sigma_{y}^{2}$ (dashed line).

vicinity at the point where the thermomechanical loading is applied. A "new" phase $(z \neq 0)$ develops near the left end of the bar where the load was applied: from $x=0$ to about $8 \times 10^{-1} \mathrm{~mm}$ in depth (see Figure 6(a)). At the end of the bar to about $2 \times 10^{-1} \mathrm{~mm}$ (resp., $10^{-1} \mathrm{~mm}$ ) with $\sigma_{y}(T, \mathbf{q})=\sigma_{y}^{1}$ (resp., $\left.\sigma_{y}^{2}\right)$, a full phase transformation $(z=1)$ developed and there existed only one phase: the daughter phase (martensite). Beyond that depth, down to $8 \times 10^{-1} \mathrm{~mm}$, the partial transformation $(z<1)$ persisted, that is, the material was a mixture between the parent phase (ferrite pearlite) and the daughter phase (martensite). On the other hand, the temporal $z$-evolution (see Figure 6(b)) shows that at time $8.5 \times 10^{-2} \leq t \leq 9.6 \times 10^{-2} \mathrm{~s}$ (resp., $8.7 \times 10^{-2} \leq t \leq 9.8 \times 10^{-2}$ s) with $\sigma_{y}(T, \mathbf{q})=\sigma_{y}^{1}\left(\right.$ resp., $\left.\sigma_{y}^{2}\right)$, the solid-solid phase transformation continued from $z=0$ until $z=1$.

The distribution of the metallurgical variable $z$ is plotted in Figure 7(a) (here only for $\sigma_{y}(T, \mathbf{q})=\sigma_{y}^{1}$ ) for three different times: $t=\left\{9 \times 10^{-2} \mathrm{~s} ; 9.5 \times 10^{-2} \mathrm{~s} ; 10^{-1} \mathrm{~s}\right\}$. It can be seen here that the irreversible metallurgical transformation was still confined near the left end of the bar, where the thermomechanical loading was applied. Figure $7(\mathrm{~b})$ shows the distribution of the temperature in the bar for the same times as previously. The nonstationary effects in the heat (8) 


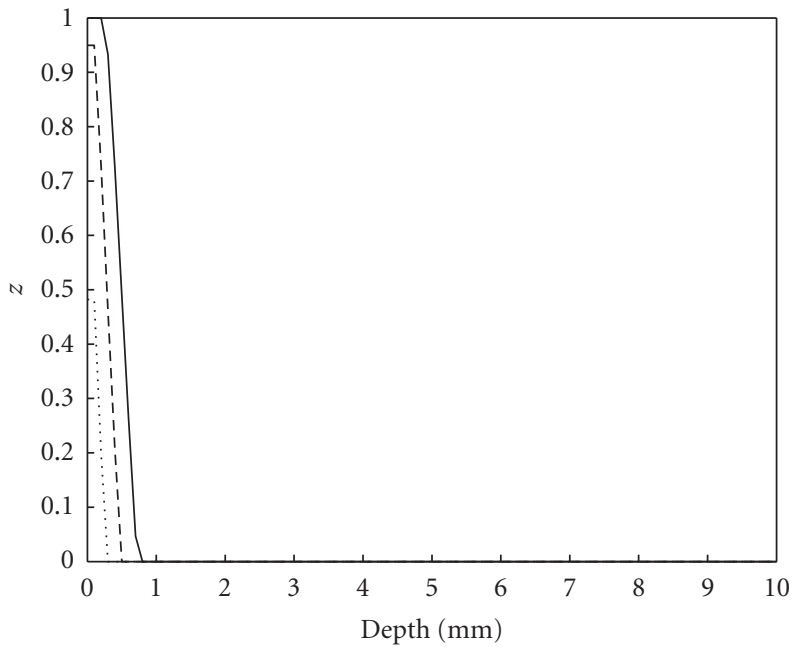

(a)

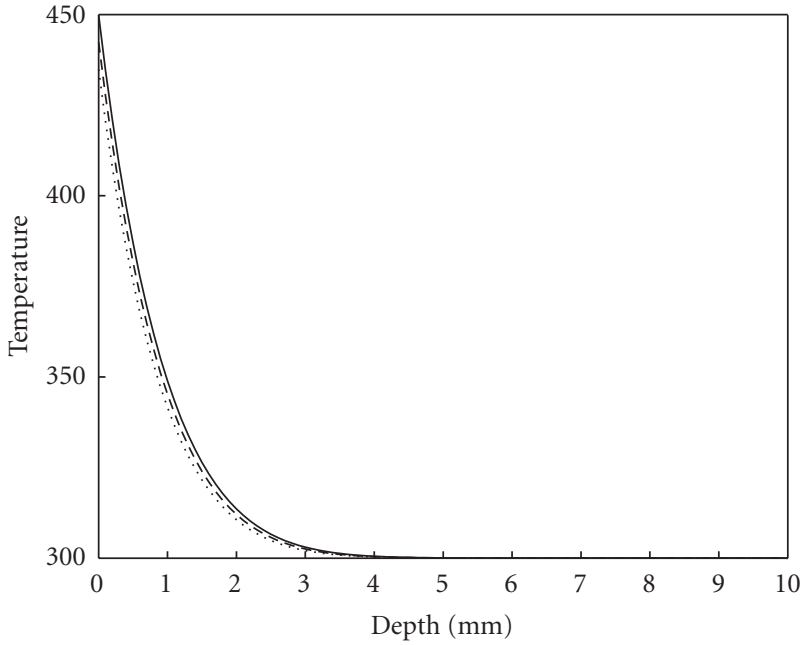

(b)

FIgURE 7: (a) Distribution of the metallurgical variable $z$ in the bar at time $t=9 \times 10^{-2} \mathrm{~s}$ (dotted line), $t=9.5 \times 10^{-2} \mathrm{~s}($ dashed line), and $t=t_{0}=10^{-1} \mathrm{~s}$ (solid line) where $\sigma_{y}(T, \mathbf{q})=\sigma_{y}^{1}$. (b) Distribution of the temperature in the bar at time $t=9 \times 10^{-2} \mathrm{~s}(\mathrm{dotted}$ line), $t=9.5 \times 10^{-2} \mathrm{~s}$ (dashed line), and $t=t_{0}=10^{-1} \mathrm{~s}$ (solid line) where $\sigma_{y}(T, \mathbf{q})=\sigma_{y}^{1}$.

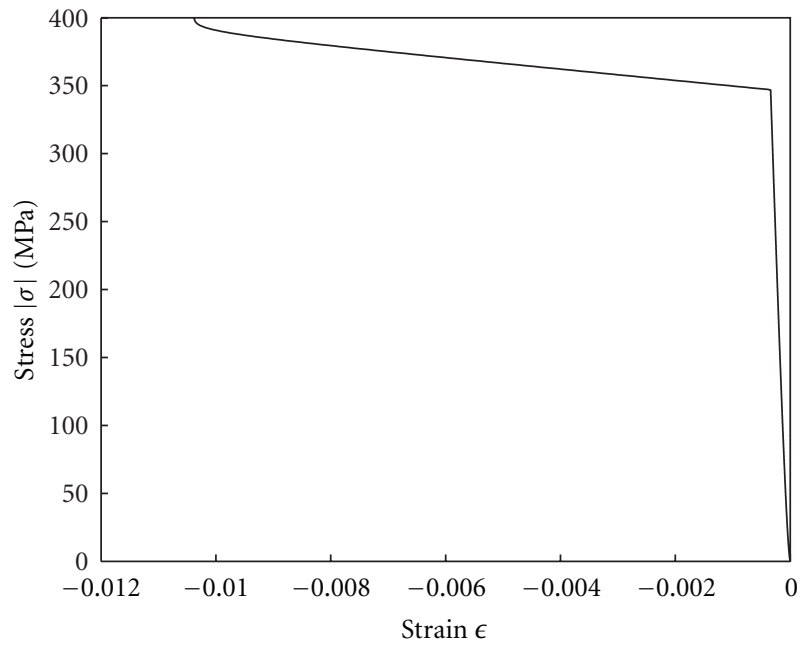

FIGURE 8: Stress-strain curve in $x=L / 100$ at time $t=t_{0}=10^{-1} \mathrm{~s}$ (in this case, only in the case of $\sigma_{y}(T, \mathbf{q})=\sigma_{y}^{1}$ ).

explain the nonlinear temperature distribution observed in the bar at the time considered.

The stress-strain curve is plotted in Figure 8 (in this case, only in the case of $\left.\sigma_{y}(T, \mathbf{q})=\sigma_{y}^{1}\right)$. The response of the material is thermoelastic when $0 \leq|\sigma| \leq 340 \mathrm{MPa}$, and inelastic behavior due to the irreversible solid-solid transformation then occurs when $340<|\sigma| \leq 384 \mathrm{MPa}$ and lastly, a return to the thermoelastic response of the material occurs when $384<|\sigma| \leq 400 \mathrm{MPa}$.

The kinetics of the transformation process presented in Section 2 describe the initiation and development of TSTs in the immediate vicinity of the surface, where the thermomechanical loading was applied. In agreement with the experimental data available on TSTs in railway tracks [8], the results presented in this Section correspond to a realistic TST thickness, that is, at a depth of around one hundred micrometers. Therefore, this metallurgical behavior model will be tested on the wheel/rail problem in the future works.

\section{Conclusion}

The kinetic model presented here describes the initiation and development of Tribological Surface Transformations (TSTs) near the surface of several rails used to railway tracks. This model is based on the assumption that the thermomechanical state in the close vicinity of the wheel/rail contact area can generate TSTs. In agreement with experimental data on TSTs, the results obtained in the case of an illustrative example show the occurrence of a completely irreversible solid-solid phase transformation in the immediate vicinity of the surface at which the thermomechanical load was applied.

\section{References}

[1] Y. Jirásková, J. Svoboda, O. Schneeweiss, W. Daves, and F. D. Fischer, "Microscopic investigation of surface layers on rails," Applied Surface Science, vol. 239, no. 2, pp. 132-141, 2005.

[2] A. Pyzalla, L. Wang, E. Wild, and T. Wroblewski, "Changes in microstructure, texture and residual stresses on the surface of a rail resulting from friction and wear," Wear, vol. 250-251, no. 2, pp. 901-907, 2001.

[3] L. Wang, A. Pyzalla, W. Stadlbauer, and E. A. Werner, "Microstructure features on rolling surfaces of railway rails subjected to heavy loading," Materials Science and Engineering A, vol. 359, no. 1-2, pp. 31-43, 2003.

[4] H. W. Zhang, S. Ohsaki, S. Mitao, M. Ohnuma, and K. Hono, "Microstructural investigation of white etching layer on pearlite steel rail," Materials Science and Engineering A, vol. 421, no. 1-2, pp. 191-199, 2006. 
[5] M. Hiensch, J. C. O. Nielsen, and E. Verheijen, "Rail corrugation in the Netherlands-measurements and simulations," Wear, vol. 253, no. 1-2, pp. 140-149, 2002.

[6] H. G. Feller and K. Walf, "Surface analysis of corrugated rail treads," Wear, vol. 144, no. 1-2, pp. 153-161, 1991.

[7] A. Saulot, Analyse tribologique du contact roue-rail-modélisation exérimentations [Ph.D. thesis], INSA Lyon, Lyon, France, 2005.

[8] W. Österle, H. Rooch, A. Pyzalla, and L. Wang, "Investigation of white etching layers on rails by optical microscopy, electron microscopy, X-ray and synchroton X-ray diffraction," Materials Science and Engineering A, vol. 303, no. 1-2, pp. 150157, 2001.

[9] E. Wild, L. Wang, B. Hasse, T. Wroblewski, G. Goerigk, and A. Pyzalla, "Microstructure alterations at the surface of a heavily corrugated rail with strong ripple formation," Wear, vol. 254, no. 9, pp. 876-883, 2003.

[10] Revue Générale des Chemins de Fer, Editions Delville, no. 144, 2005.

[11] R. I. Carroll and J. H. Beynon, "Rolling contact fatigue of white etching layer: part 1. Crack morphology," Wear, vol. 262, no. 9-10, pp. 1253-1266, 2007.

[12] R. I. Carroll and J. H. Beynon, "Rolling contact fatigue of white etching layer. Part 2. Numerical results," Wear, vol. 262, no. 910 , pp. 1267-1273, 2007.

[13] G. Baumann, H. J. Fecht, and S. Liebelt, "Formation of whiteetching layers on rail treads," Wear, vol. 191, no. 1-2, pp. 133 140, 1996.

[14] G. Antoni, Transformation tribologique de surface: une approche thermo-mécanique [Ph.D. thesis], Université de Provence, Marseille, France, 2010.

[15] J. Lemaitre and J. L. Chaboche, Mécanique des Matériaux Solides, Dunod, Paris, France, 1996. 

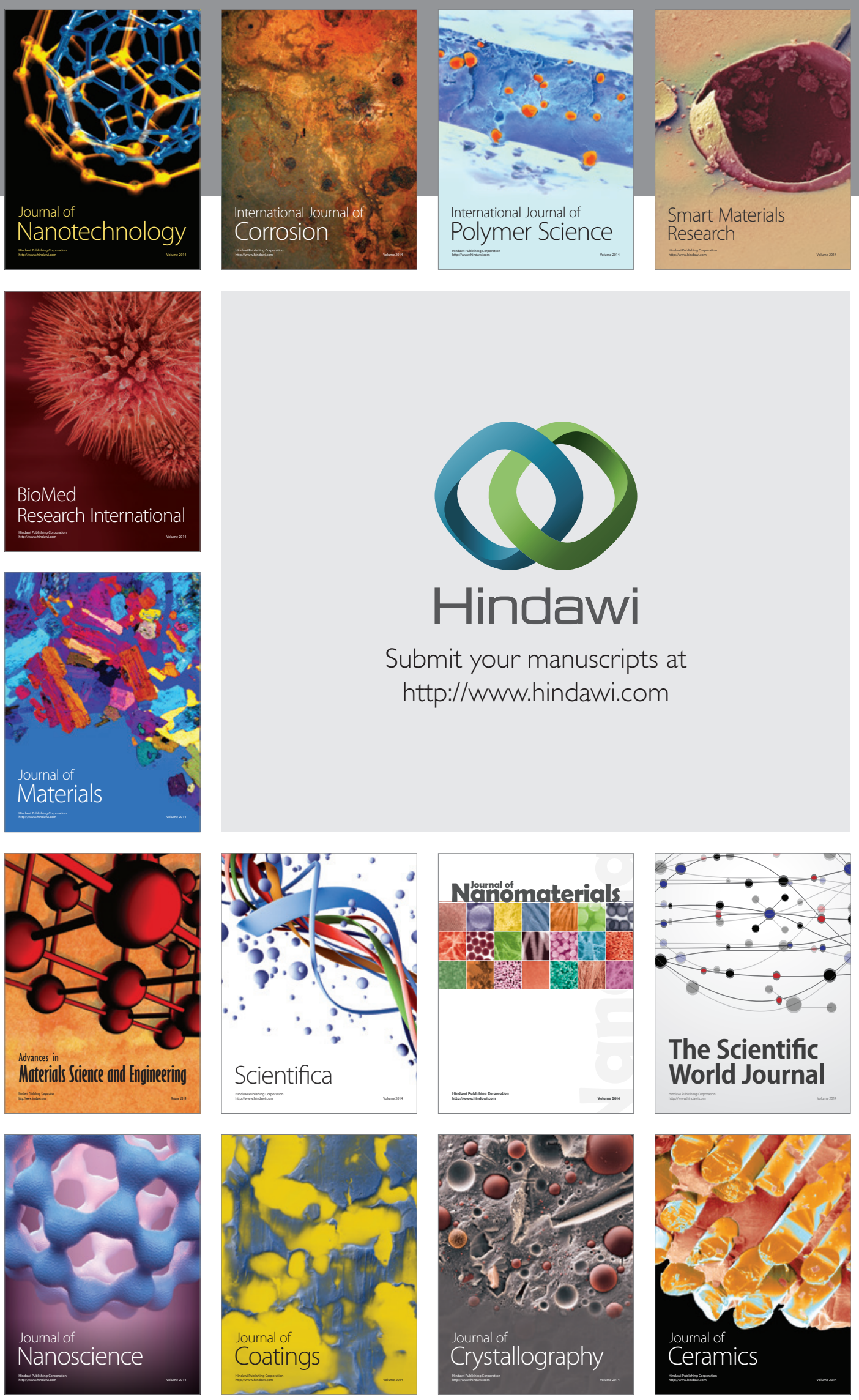

The Scientific World Journal

Submit your manuscripts at

http://www.hindawi.com

\section{World Journal}

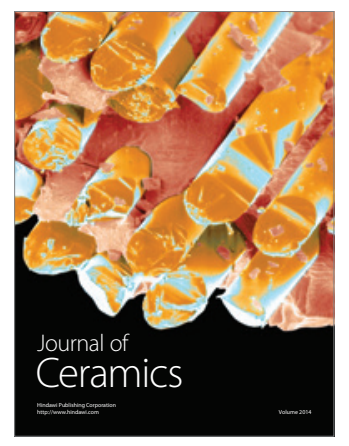

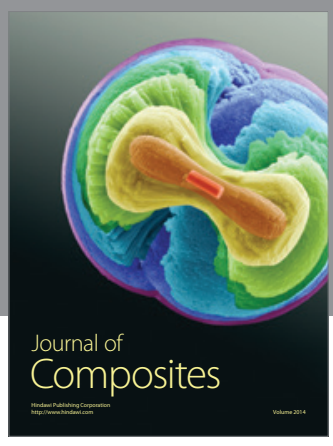
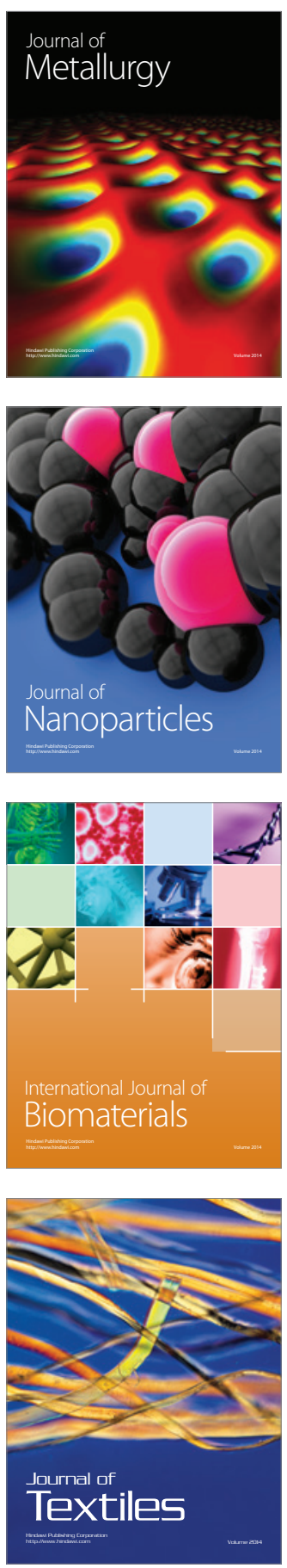